\section{Ocular Onchocerca sp infection in a dog - a case report and review of the literature}

\section{Sasja Borup Pustelnik, Raquel Salguero, David Williams}

Department of Veterinary Medicine, Cambridge University, Cambridge, UK

\section{OBJECTIVES}

Description of ocular infection with Onchocerca lupi in the United Kingdom

\section{METHODS}

Reports of infection with Onchocerca lupi nematodes generally originate from areas of Greece and Portugal. The increasing importation of rescued stray animals from these areas may result in an increase in cases seen in the UK. Here we document one such case.

\section{RESULTS}

A 5-year old male neutered German shepherd crossbreed dog imported as a rescue from Romania was referred for investigation of a $1 \times 0.5 \mathrm{~cm}$ periocular subconjunctival mass. Ophthalmic examination showed no other ocular lesions. Intraocular pressure was lower in the affected eye than the normal, suggesting mild intraocular inflammation. Vision was deemed to be unaffected given the dog's normal behaviour and a positive menace and pupillary light response. Ultrasound examination of the globe revealed several hyperechoic curvilinear small bodies within a thin-walled subconjunctival cystic structure. A CT scan was performed and confirmed the presence of a cyst with small hyperdense structures. Resection of the mass was achieved uneventfully under general anaesthesia and small nematode parasites were seen on opening the mass. Histological examination of the lesion confirmed the presence of adult nematodes and an entire nematode removed was identified as Onchocerca lupi on microscopy. The dog was treated with ivermectin at $50 \mathrm{ug} / \mathrm{kg}$ subcutaneously. No recurrence was noted up to 18 months post-operatively.

\section{STATEMENT}

Onchocerca lupi is a canine parasite of zoonotic concern, and this case serves to demonstrate that periocular masses noted in dogs imported from areas in which the parasite is endemic should be treated with caution.

\section{Canine ocular dermoids: a case series}

\section{David Williams, Owain Davies}

Department of Veterinary Medicine, University of Cambridge, Cambridge, Cambs, UK

\section{OBJECTIVES}

To document a case series of ocular dermoids in dogs and to determine potential breed predisposition for the condition.

\section{METHODS}

A retrospective case study was conducted of ocular dermoids in canine patients from centres in the UK, Germany and North America.

\section{RESULTS}

111 ocular surface dermoids in 96 patients were documented over 10 years, 26 cases at the Queen's Veterinary
School Hospital, Cambridge,13 from the Free Univeristat, Berlin and 57 from the Comparative Ocular Pathology Laboratory, Wisconsin, USA. The most common breeds were the Shih Tzu (15 dogs, 17\% of cases), French Bulldogs (11 dogs 13\% of cases), German shepherd dogs ( 8 dogs, $9 \%$ of cases), Rottweilers ( 7 dogs, $8 \%$ of cases), Labrador Retrievers (7 dogs, $8 \%$ of cases) and Cavalier King Charles Spaniels (CKCS) (5 dogs 6\% of cases). $45 \%$ were female and $55 \%$ male. 40 lesions were corneal, 29 conjunctival, 12 involving cornea and conjunctiva, 3 were palpebral and 1 involved conjunctiva and eyelid. 11 dogs exhibited bilateral dermoids. Mann's categorization of dermoids as type 1 (limbal or epibulbar), type 2 (corneal) or type 3 involving the entirety of the anterior segment. Most canine dermoids were type 1 with a smaller number being type 2 and no type 3 in this case series. All lesions were subjected to surgical resection and histology demonstrating the lesion to be dermal in nature.

\section{STATEMENT}

This study provides the largest case series of ocular dermoids in the veterinary literature, demonstrating breed predispositions for the condition in shit tzus and French bulldogs. 